\title{
Learning Relational Dependency Networks for Relation Extraction
}

\author{
Dileep Viswanathan \\ School of Informatics and Computing \\ Indiana University \\ diviswan@indiana.edu \\ Jude Shavlik \\ Department of Computer Sciences \\ University of Wisconsin - Madison \\ shavlik@cs.wisc.edu
}

\section{Abstract}

We consider the task of KBP slot filling - extracting relation information from newswire documents for knowledge base construction. We present our pipeline, which employs Relational Dependency Networks (RDNs) to learn linguistic patterns for relation extraction. Additionally, we demonstrate how several components such as weak supervision, word2 vec features, joint learning and the use of human advice, can be incorporated in this relational framework. We evaluate the different components in the benchmark KBP 2015 task and show that RDNs effectively model a diverse set of features and perform competitively with current state-of-the-art relation extraction.

\section{Introduction}

The problem of knowledge base population (KBP) - constructing a knowledge base (KB) of facts gleaned from a large corpus of unstructured data - poses several challenges for the NLP community. Commonly, this relation extraction task is decomposed into two subtasks - entity linking, in which entities are linked to already identified identities within the document or to entities in the existing $\mathrm{KB}$, and slot filling, which identifies certain attributes about a target entity.

We present our work-in-progress for KBP slot filling based on our probabilistic logic formalisms and present the different components of the system. Specifically, we employ Relational Dependency Networks (Neville and Jensen 2007), a formalism that has been successfully used for joint learning and inference from stochastic, noisy, relational data. We consider our RDN system against the current stateof-the-art for KBP to demonstrate the effectiveness of our probabilistic relational framework.

Additionally, we show how RDNs can effectively incorporate many popular approaches in relation extraction such as joint learning, weak supervision, word2vec features, and human advice, among others. We provide a comprehensive comparison of settings such as joint learning vs learning of individual relations, use of weak supervision vs gold standard labels, using expert advice vs only learning from data, etc. These questions are extremely interesting from a general machine learning perspective, but also critical to the NLP community. As we show empirically, some of the re-

\section{Ameet Soni}

Department of Computer Science

Swarthmore College

sonidcs.swarthmore.edu

\author{
Sriraam Natarajan \\ School of Informatics and Computing \\ Indiana University \\ natarasr@indiana.edu
}

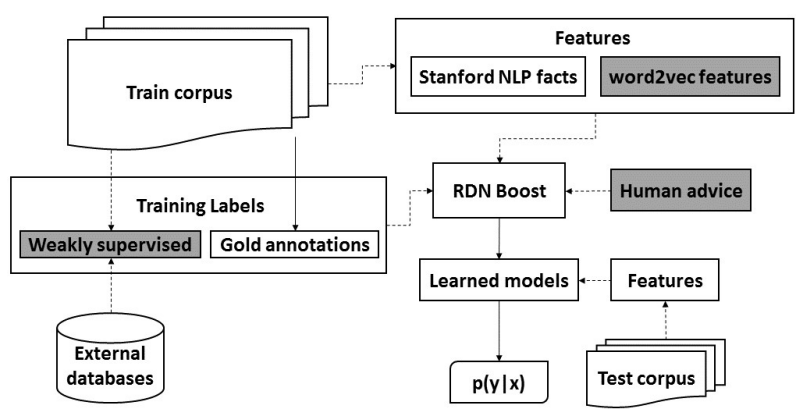

Figure 1: Pipeline Full RDN relation extraction pipeline. Components in shaded boxes indicate proposed contributions in this work for (1) adding training labels (weak supervision), (2) enhancing feature descriptors (word2vec), and (3) initializing the RDN with human advice rules.

sults such as human advice being useful in many relations and joint learning being beneficial in the cases where the relations are correlated among themselves are on the expected lines. However, some surprising observations include the fact that weak supervision is not as useful as expected and word2vec features are not as predictive as the other domain-specific features.

We first present the proposed pipeline with all the different components of the learning system. Next we present the set of 14 relations that we learn on before presenting the experimental results. We finally discuss the results of these comparisons before concluding by presenting directions for future research.

\section{Proposed Pipeline}

We present the different aspects of our pipeline, depicted in Figure 1. We will first describe our approach to generating features and training examples from the KBP corpus, before describing the core of our framework - the RDN Boost algorithm.

\section{Feature Generation}

Given a training corpus of raw text documents, our learning algorithm first converts these documents into a set of facts (i.e., features) that are encoded in first order logic 


\begin{tabular}{|l|l|}
\hline Feature & Description \\
\hline $\begin{array}{l}\text { wordString } \\
\text { cordPosition } \\
\text { wordLessWordString }\end{array}$ & $\begin{array}{l}\text { word with word id } \\
\text { location of the word } \\
\text { word string in lower case } \\
\text { canonical form of word }\end{array}$ \\
isNEWord & whether word is NE \\
nextWords & two succeeding words \\
two preceding words \\
nextPOS & POS for the succeeding words \\
prevPOS & POS for the preceding words \\
nextLemmas & canonical form of successors \\
prevLemmas & canonical form of predecessors \\
nextNE & succeeding NE phrases \\
prevNE & preceding NE phrases \\
lemmaBetween & canonical form of word \\
occurring between two NEs & word b/w two NEs is an NE \\
neBetween & POS of word b/w two NEs \\
posBetween & \\
Dependency Path & \\
rootChildLemma & canonical form of child of DPR \\
rootChildNER & child of DPR is NE \\
rootChildPOS & POS of child of DPR \\
rootLemma & lemma of DPR \\
rootNER & DPR is NER \\
rootPOS & POS of DPR \\
\hline
\end{tabular}

Table 1: Standard NLP Features Features derived from the training corpus used by our learning system. POS - part of speech. NE - Named Entity. DPR - root of dependency path tree.

(FOL). Raw text is processed using the Stanford CoreNLP Toolki 1 (Manning et al. 2014) to extract parts-of-speech, word lemmas, etc. as well as generate parse trees, dependency graphs and named-entity recognition information. The full set of extracted features is listed in Table 1 . These are then converted into features in prolog (i.e., FOL) format and are given as input to the system.

In addition to the structured features from the output of Stanford toolkit, we also use deeper features based on word2vec (Mikolov et al. 2013) as input to our learning system. Standard NLP features tend to treat words as individual objects, ignoring links between words that occur with similar meanings or, importantly, similar contexts (e.g., city-country pairs such as Paris - France and Rome - Italy occur in similar contexts). word2 vec provide a continuous-space vector embedding of words that, in practice, capture many of these relationships (Mikolov et al. 2013, Mikolov, Yih, and Zweig 2013). We use word vectors from Stanford ${ }^{2}$ and Google ${ }^{3}$ along with a few specific words that, experts believe, are related to the relations learned. For example, we include words such as "father" and "mother" (inspired by the parent relation) or "devout","convert", and "follow" (religion relation). We generated features from word vectors by finding words with high similarity in the embedded space. That is, we used word vectors by considering relations of the following

\footnotetext{
1http://stanfordnlp.github.io/CorenLP /

2 http://nlp.stanford.edu/projects/glove/ 3thps://code.google.com/p/word2vec/
}

form: similar $W$ ords(wordA, wordB, $\max S i m)$, where maxSim is the cosine similarity score between the words. Only the top cosine similarity scores for a word are utilized.

\section{Weak Supervision}

One difficulty with the KBP task is that very few documents come labeled as gold standard labels, and further annotation is prohibitively expensive beyond a few hundred documents. This is problematic for discriminative learning algorithms, like the RDN learning algorithm, which excel when given a large supervised training corpus. To overcome this obstacle, we employ weak supervision - the use of external knowledge (e.g., a database) to heuristically label examples. Following our work in Soni et al. (2016), we employ two approaches for generating weakly supervised examples - distant supervision and knowledge-based weak supervision.

Distant supervision entails the use of external knowledge (e.g., a database) to heuristically label examples. Following standard procedure, we use three data sources Never Ending Language Learner (NELL) (Carlson et al. 2010), Wikipedia Infoboxes and Freebase. For a given target relation, we identify relevant database(s), where the entries in the database form entity pairs (e.g., an entry of (Barack Obama, Malia Obama) for a parent database) that will serve as a seed for positive training examples. These pairs must then be mapped to mentions in our corpus - that is, we must find sentences in our corpus that contain both entities together (Zhang et al. 2012). This process is done heuristically and is fraught with potential errors and noise (Riedel, Yao, and McCallum 2010).

An alternative approach, knowledge-based weak supervision is based on previous work (Natarajan et al. 2014; Soni et al. 2016) with the following insight: labels are typically created by "domain experts" who annotate the labels carefully, and who typically employ some inherent rules in their mind to create examples. For example, when identifying family relationship, we may have an inductive bias towards believing two persons in a sentence with the same last name are related, or that the words "son" or "daughter" are strong indicators of a parent relation. We call this world knowledge as it describes the domain (or the world) of the target relation.

To this effect, we encode the domain expert's knowledge in the form of first-order logic rules with accompanying weights to indicate the expert's confidence. We use the probabilistic logic formalism Markov Logic Networks (Domingos and Lowd 2009) to perform inference on unlabeled text (e.g., the TAC KBP corpus). Potential entity pairs from the corpus are queried to the MLN, yielding (weaklysupervised) positive examples. We choose MLNs as they permit domain experts to easily write rules while providing a probabilistic framework that can handle noise, uncertainty, and preferences while simultaneously ranking positive examples.

We use the Tuffy system (Niu et al. 2011) to perform inference. The inference algorithm implemented inside Tuffy appears to be robust and scales well to millions of docu- 


\begin{tabular}{|c|l|}
\hline Weight & MLN Clause \\
\hline 1.0 & entityType(a, "PER"), entityType(b, "NUM"), nextWord(a, c), word(c, “,"), \\
& nextWord(c, b) $\rightarrow$ age(a, b) \\
0.6 & entityType(a, "PER"), entityType(b, "NUM"), prevLemma(b, “age") $\rightarrow$ age (a, b) \\
0.8 & entityType(a, "PER"), entityType(b, "PER"), nextLemma(a, "mother") $\rightarrow$ parents(a, b) \\
0.8 & entityType(a, "PER"), entityType(b, "PER"), nextLemma(a, "father") $\rightarrow$ parents(a, b) \\
\hline
\end{tabular}

Table 2: Rules for KB Weak Supervision A sample of knowledge-based rules for weak supervision provided by labelers. The first value defines a weight, or confidence in the accuracy of the rule. The target relation appears at the end of each clause. "PER", "ORG", "NUM" represent entities that are persons, organizations, and numbers, respectively.

ments 4

For the KBP task, some rules that we used are shown in Table 2 For example, the first rule identifies any number following a person's name and separated by a comma is likely to be the person's age (e.g., "Sharon, 42"). The third and fourth rule provide examples of rules that utilize more textual features; these rules state the appearance of the lemma "mother" or "father" between two persons is indicative of a parent relationship (e.g.,"Malia's father, Barack, introduced her...”).

\section{Learning Relational Dependency Networks}

Previous research (Meza-Ruiz and Riedel 2009) has demonstrated that joint inferences of the relations are more effective than considering each relation individually. Consequently, we have considered a formalism that has been successfully used for joint learning and inference from stochastic, noisy, relational data called Relational Dependency Networks (RDNs) (Neville and Jensen 2007, Natarajan et al. 2010). RDNs extend dependency networks (DN) (Heckerman et al. 2001) to the relational setting. The key idea in a $\mathrm{DN}$ is to approximate the joint distribution over a set of random variables as a product of their marginal distributions, i.e., $P\left(y_{1}, \ldots, y_{n} \mid \mathbf{X}\right) \approx \prod_{i} P\left(y_{i} \mid \mathbf{X}\right)$. It has been shown that employing Gibbs sampling in the presence of a large amount of data allows this approximation to be particularly effective. Note that, one does not have to explicitly check for acyclicity making these DNs particularly easy to be learned.

In an RDN, typically, each distribution is represented by a relational probability tree (RPT) (Neville et al. 2003). However, following previous work (Natarajan et al. 2010), we replace the RPT of each distribution with a set of relational regression trees (Blockeel and Raedt 1998) built in a sequential manner i.e., replace a single tree with a set of gradient boosted trees. This approach has been shown to have stateof-the-art results in learning RDNs and we adapted boosting to learn for relation extraction. Since this method requires negative examples, we created negative examples by considering all possible combinations of entities that are not present in positive example set and sampled twice as many negatives as positive examples.

\footnotetext{
${ }^{4}$ As the structure and weights are pre-defined by the expert, learning is not needed for our MLN
}

\begin{tabular}{|l|}
\hline Advice Rules \\
\hline $\begin{array}{l}\text { Entity preceded by a number and a phrase "year-old" } \\
\text { probably refers to age. }\end{array}$ \\
\hline $\begin{array}{l}\text { Entity present with a phrase in sentence "who turned" } \\
\text { probably refers to age. }\end{array}$ \\
\hline $\begin{array}{l}\text { Entity1 is "also known as" Entity2 } \\
\text { probably refers to alternate name. }\end{array}$ \\
\hline $\begin{array}{l}\text { Entity1, "nicknamed" Entity2 } \\
\text { probably refers to alternate name. }\end{array}$ \\
\hline $\begin{array}{l}\text { Entity1 followed by phrase "is a citizen of" Entity2 } \\
\text { probably refers to origin. }\end{array}$ \\
\hline $\begin{array}{l}\text { Entity followed by phrase "is a devout" Entity2 } \\
\text { probably refers to religion. }\end{array}$ \\
\hline $\begin{array}{l}\text { Entity, followed by "a" Entity2"-based company" } \\
\text { probably refers to city/state/country of headquarters. }\end{array}$ \\
\hline $\begin{array}{l}\text { If Entity1 and Entity2 are siblings } \\
\text { then they are not parents of each other. }\end{array}$ \\
\hline $\begin{array}{l}\text { If Entity1 and Entity2 are spouses of each other } \\
\text { then they are not parents of each other. }\end{array}$ \\
\hline
\end{tabular}

Table 3: Advice Rules Sample advice rules used for relation extraction. We employed a total of 72 such rules for our 14 relations.

\section{Incorporating Human Advice}

While most relational learning methods restrict the human to merely annotating the data, we go beyond and request the human for advice. The intuition is that we as humans read certain patterns and use them to deduce the nature of the relation between two entities present in the text. The goal of our work is to capture such mental patterns of the humans as advice to the learning algorithm. We modified the work of Odom et al. (2015a; 2015b) to learn RDNs in the presence of advice. The key idea is to explicitly represent advice in calculating gradients. This allows the system to trade-off between data and advice throughout the learning phase, rather than only consider advice in initial iterations. Advice, in particular, become influential in the presence of noisy or less amout of data.

A few sample advice rules in English (these are converted to first-order logic format and given as input to our algorithm) are presented in Table 3 . Note that some of the rules are "soft" rules in that they are not true in many situations. Odom et al. (2015b) weigh the effect of the rules against the data and hence allow for partially correct rules. 


\begin{tabular}{|c|c|c|c|}
\hline Relation & Gold & WS & Test \\
\hline per: age & 89 & 150 & 44 \\
\hline per : alternateName & 28 & $\mathrm{x}$ & 18 \\
\hline per : children & 89 & $\mathrm{X}$ & 23 \\
\hline per : origin & 96 & 150 & 48 \\
\hline per : otherFamily & 72 & 150 & 10 \\
\hline per : parents & 71 & 150 & 30 \\
\hline per : religion & 70 & 150 & 11 \\
\hline per : siblings & 77 & 150 & 31 \\
\hline per : spouse & 66 & 150 & 28 \\
\hline per : title & 158 & $\mathrm{x}$ & 39 \\
\hline org : city $H Q$ & 69 & $\mathrm{X}$ & 10 \\
\hline org : countryHQ & 69 & 150 & 29 \\
\hline org : dateFounded & 70 & 150 & 17 \\
\hline org : foundedBy & 62 & 150 & 32 \\
\hline
\end{tabular}

Table 4: Relations The set of relations considered from TAC KBP. Columns indicate the number of training examples utilized - both human annotated (Gold) and weakly supervised (WS), when available - from TAC KBP 2014 and number of test examples from TAC KBP 2015. 10 relations describe person entities (per) while the last 4 describe organizations (org).

\section{Experiments and Results}

We now present our experimental evaluation. We considered 14 specific relations from two categories, person and organization from the TAC KBP competition. The relations considered are listed in the left column of Table 4. We utilize documents from KBP 2014 for training while utilizing documents from the 2015 corpus for testing.

All results presented are obtained from 5 different runs of the train and test sets to provide more robust estimates of accuracy. We consider three standard metrics - area under the ROC curve, F-1 score and the recall at a certain precision. We chose the precision as 0.66 since the fraction of positive examples to negatives is 1:2 (we sub-sampled the negative examples for the different training sets). Negative examples are re-sampled for each training run. It must be mentioned that not all relations had the same number of hand-annotated (gold standard) examples because the 781 documents that we annotated had different number of instances for these relations. The train/test gold-standard sizes are provided in the table, including weakly supervised examples, if available. Lastly, to control for other factors, the default setting for our experiments is individual learning, standard features, with gold standard examples only (i.e., no weak supervision, word2 vec, advice, or advice).

Since our system had different components, we aimed to answer the following questions:

Q1: Do weakly supervised examples help construct better models?

Q2: Does joint learning help in some relations?

Q3: Are word2vec features more predictive than standard features presented in Table 1 ?

Q4: Does advice improve performance compared to just learning from data?

\begin{tabular}{|l|cc|cc|}
\hline Relation & \multicolumn{2}{|c|}{ AUC ROC } & \multicolumn{2}{c|}{ F1 } \\
& $\mathrm{G}$ & $\mathrm{G}+\mathrm{WS}$ & $\mathrm{G}$ & $\mathrm{G}+\mathrm{W}$ \\
\hline age & 0.90 & 0.93 & 0.64 & 0.76 \\
origin & 0.74 & 0.81 & 0.12 & 0.06 \\
otherFamily & 0.78 & 0.83 & 0.13 & 0.23 \\
parents & 0.69 & 0.62 & 0.13 & 0.25 \\
religion & 0.77 & 0.73 & 0.64 & 0.54 \\
siblings & 0.81 & 0.77 & 0.19 & 0.19 \\
spouse & 0.83 & 0.86 & 0.31 & 0.33 \\
countryHQ & 0.77 & 0.75 & 0.41 & 0.42 \\
dateFounded & 0.88 & 0.86 & 0.43 & 0.50 \\
foundedBy & 0.85 & 0.77 & 0.50 & 0.37 \\
\hline
\end{tabular}

Table 5: Weak Supervision Results comparing models trained with gold standard examples only $(\mathrm{G})$ and models trained with gold standard and weakly supervised examples combined (G+WS).

Q5: How does our system, that includes all the components, perform against a robust baseline (Relation Factory (Roth et al. 2014))?

\section{Weak Supervision}

To answer Q1, we generated positive training examples using the weak supervision techniques specified earlier. Specifically, we evaluated 10 relations as show in Table 5 Based on experiments from (Soni et al. 2016), we utilized our knowledge-based weak supervision approach to provide positive examples in all but two of our relations. A range of 4 to 8 rules are derived for each relation. Examples for the organization relations countryHQ and foundedBy were generated using standard distant supervision techniques Freebase databases were mapped to foundedBy while Wikipedia Infoboxes provides entity pairs for country $H Q$. Lastly, only 150 weakly supervised examples were utilized in our experiments (all gold standard examples were utilized). Performing larger runs is part of work in progress.

The results are presented in Table 5. We compared our standard pipeline (individually learned relations with only standard features) learned on gold standard examples only versus our system learned with weak and gold examples combined. Surprisingly, weak supervision does not seem to help learn better models for inferring relations in most cases. Only two relations - origin, other Family - see substantial improvements in AUC ROC, while F1 shows improvements for age and, other Family, and dateFounded. We hypothesize that generating more examples will help (some relations produced thousands of examples), but nonetheless find the lack of improved models from even a modest number of examples a surprising result. Alternatively, the number of gold standard examples provided may be sufficient to learn RDN models. Thus Q1 is answered equivocally, but in the negative.

\section{Joint learning}

To address our next question, we assessed our pipeline when learning relations independently (i.e., individually) versus 


\begin{tabular}{|l|cc|}
\hline Relation & \multicolumn{2}{|c|}{ AUC ROC } \\
& IL & JL \\
\hline age & 0.93 & 0.93 \\
alternateName & 0.91 & 0.75 \\
children & 0.75 & 0.76 \\
origin & 0.86 & 0.89 \\
otherFamily & 0.88 & 0.89 \\
parents & 0.74 & 0.74 \\
religion & 0.72 & 0.79 \\
siblings & 0.79 & 0.80 \\
spouse & 0.86 & 0.87 \\
title & 0.90 & 0.89 \\
cityHQ & 0.74 & 0.73 \\
countryHQ & 0.75 & 0.79 \\
dateFounded & 0.87 & 0.86 \\
foundedBy & 0.83 & 0.86 \\
\hline
\end{tabular}

Table 6: Joint Learning Results comparing models trained individually (IL) and models trained with jointly for all relations (JL).

learning relations jointly within the RDN, displayed in Table 6 . Recall and F1 are omitted for conciseness - the conclusions are the same across all metrics. Joint learning appears to help in about half of the relations (8/14). Particularly, in person category, joint learning with gold standard outperforms their individual learning counterparts. This is due to the fact that some relations such as parents, spouse, siblings etc. are inter-related and learning them jointly indeed improves performance. Hence Q2 can be answered affirmatively for half the relations.

\section{word2vec}

Table 7 shows the results of experiments comparing the RDN framework with and without word2vec features. word2 vec appears to largely have no impact, boosting results in just 4 relations. We hypothesize that this may be due to a limitation in the depth of trees learned. Learning more and/or deeper trees may improve use of word2vec features, and additional work can be done to generate deep features from word vectors. Q3 is answered cautiously in the negative, although future work could lead to improvements.

\section{Advice}

Table 8 shows the results of experiments that test the use of advice within the joint learning setting. The use of advice improves or matches the performance of using only joint learning. The key impact of advice can be mostly seen in the improvement of recall in several relations. This clearly shows that using human advice patterns allows us to extract more relations effectively making up for noisy or less number of training examples. This is in-line with previously published machine learning literature (Towell and Shavlik 1994, Fung, Mangasarian, and Shavlik 2002; Kunapuli et al. 2013, Odom et al. 2015b) in that humans can be more than mere labelers by providing useful advice to learning algorithms that can improve their performance. Thus Q4 can be answered affirmatively.

\begin{tabular}{|l|cc|}
\hline Relation & \multicolumn{2}{|c|}{ AUC ROC } \\
& $-\mathrm{w} 2 \mathrm{v}$ & $+\mathrm{w} 2 \mathrm{v}$ \\
\hline age & 0.93 & 0.91 \\
alternateName & 0.75 & 0.73 \\
children & 0.76 & 0.79 \\
origin & 0.89 & 0.90 \\
otherFamily & 0.89 & 0.78 \\
parents & 0.74 & 0.70 \\
religion & 0.79 & 0.74 \\
siblings & 0.80 & 0.75 \\
spouse & 0.87 & 0.83 \\
title & 0.89 & 0.90 \\
cityHQ & 0.73 & 0.73 \\
countryHQ & 0.79 & 0.78 \\
dateFounded & 0.86 & 0.84 \\
foundedBy & 0.86 & 0.94 \\
\hline
\end{tabular}

Table 7: word2vec Results comparing models trained without $(-w 2 v)$ and with word2vec features $(+w 2 v)$.

\begin{tabular}{|l|cc|cc|}
\hline Relation & \multicolumn{2}{|c|}{ AUC ROC } & \multicolumn{2}{c|}{ Recall } \\
& -Adv & +Adv & -Adv & +Adv \\
\hline age & 0.93 & 0.93 & 0.56 & 0.74 \\
alternateName & 0.75 & 0.77 & 0.20 & 0.16 \\
children & 0.76 & 0.76 & 0.04 & 0.14 \\
origin & 0.89 & 0.88 & 0.86 & 0.82 \\
otherFamily & 0.89 & 0.90 & 0 & 0.06 \\
parents & 0.74 & 0.72 & 0.15 & 0.05 \\
religion & 0.79 & 0.81 & 0.51 & 0.56 \\
siblings & 0.80 & 0.81 & 0.04 & 0.00 \\
spouse & 0.87 & 0.85 & 0.06 & 0.04 \\
title & 0.89 & 0.90 & 0.16 & 0.07 \\
cityHQ & 0.73 & 0.74 & 0.26 & 0.28 \\
countryHQ & 0.79 & 0.77 & 0.61 & 0.62 \\
dateFounded & 0.86 & 0.86 & 0.20 & 0.05 \\
foundedBy & 0.86 & 0.84 & 0.24 & 0.25 \\
\hline
\end{tabular}

Table 8: Advice Results comparing models trained without $(-A d v)$ and with advice (+Adv).

\section{RDN Boost vs Relation Factory}

Relation factory (RF) (Roth et al. 2014) is an efficient, open source system for performing relation extraction based on distantly supervised classifiers. It was the top system in the TAC KBP 2013 competition (Surdeanu 2013) and thus serves as a suitable baseline for our method. RF is very conservative in its responses, making it very difficult to adjust the precision levels. To be most generous to RF, we present recall for all returned results (i.e., score $>0$ ). The AUC ROC, recall, and F1 scores of our system against RF are presented in Table 9

Our system performs comparably, and often better than the state-of-the-art Relation Factory system. In particular, our method outperforms Relation Factory in AUC ROC across all relations. Recall provides a more mixed picture with both approaches showing some improvements - RDN outperforms in 6 relations while Relation Factory does so in 


\begin{tabular}{|l|cc|cc|cc|}
\hline Relation & \multicolumn{2}{|c|}{ AUC ROC } & \multicolumn{2}{|c|}{ Recall } & \multicolumn{2}{c|}{ F1 } \\
& RF & RDN & RF & RDN & RF & RDN \\
\hline age & 0.64 & $\mathbf{0 . 9 3}$ & 0.28 & $\mathbf{0 . 7 4}$ & 0.44 & $\mathbf{0 . 6 7}$ \\
alternateName & 0.50 & $\mathbf{0 . 7 7}$ & 0.00 & $\mathbf{0 . 1 6}$ & 0 & $\mathbf{0 . 1 0}$ \\
children & 0.54 & $\mathbf{0 . 7 6}$ & 0.09 & $\mathbf{0 . 1 4}$ & 0.17 & $\mathbf{0 . 2 8}$ \\
origin & 0.50 & $\mathbf{0 . 8 9}$ & 0.00 & $\mathbf{0 . 8 6}$ & 0 & $\mathbf{0 . 6 4}$ \\
otherFamily & 0.56 & $\mathbf{0 . 9 0}$ & $\mathbf{0 . 1 1}$ & 0.06 & $\mathbf{0 . 2 4}$ & 0.22 \\
parents & 0.29 & $\mathbf{0 . 7 4}$ & $\mathbf{0 . 3 3}$ & 0.15 & $\mathbf{0 . 5 0}$ & 0.31 \\
religion & 0.50 & $\mathbf{0 . 8 1}$ & 0 & $\mathbf{0 . 5 6}$ & 0 & $\mathbf{0 . 6 0}$ \\
siblings & 0.13 & $\mathbf{0 . 8 1}$ & $\mathbf{0 . 1 7}$ & 0.00 & 0.29 & 0.29 \\
spouse & 0.57 & $\mathbf{0 . 8 5}$ & $\mathbf{0 . 1 3}$ & 0.04 & 0.23 & $\mathbf{0 . 3 7}$ \\
title & 0.67 & $\mathbf{0 . 9 0}$ & $\mathbf{0 . 6 7}$ & 0.07 & $\mathbf{0 . 8 0}$ & 0.54 \\
cityHQ & 0.38 & $\mathbf{0 . 7 4}$ & $\mathbf{0 . 3 8}$ & 0.28 & $\mathbf{0 . 5 5}$ & 0.41 \\
countryHQ & 0.57 & $\mathbf{0 . 7 7}$ & 0.14 & $\mathbf{0 . 6 2}$ & 0.25 & $\mathbf{0 . 5 8}$ \\
dateFounded & 0.67 & $\mathbf{0 . 8 6}$ & $\mathbf{0 . 3 3}$ & 0.05 & $\mathbf{0 . 5 0}$ & 0.46 \\
foundedBy & 0.20 & $\mathbf{0 . 8 4}$ & $\mathbf{0 . 3 7}$ & 0.25 & 0.54 & $\mathbf{0 . 5 5}$ \\
\hline
\end{tabular}

Table 9: Relation Factory vs RDN Results comparing Relation Factory (RF) with the RDN algorithm presented in this paper. Values in bold indicate superiour performance against the alternative approach.

8. Note that in the instances where RDN provides superior recall, it does so with dramatic improvements (RF often returns 0 positives in these relations). F1 also shows RDN's superior performance, outperforming RF in most relations. Thus, the conclusion for $\mathbf{Q 5}$ is that our RDN framework performas comparably, if not better, across all metrics against the state-of-the-art.

\section{Conclusion}

We presented our fully relational system utilizing Relational Dependency Networks for the Knowledge Base Population task. We demonstrated RDN's ability to effectively learn the relation extraction task, performing comparably (and often better) than the state-of-art Relation Factory system. Furthermore, we demonstrated the ability of RDNs to incorporate various concepts in a relational framework, including word2vec, human advice, joint learning, and weak supervision. Some surprising results are that weak supervision and word2vec did not significantly improve performance. However, advice is extremely useful thus validating the long-standing results inside the Artificial Intelligence community for the relation extraction task as well. Possible future directions include considering a larger number of relations, deeper features and finally, comparisons with more systems. We believe further work on developing word2vec features and utilizing more weak supervision examples may reveal further insights into how to effectively utilize such features in RDNs.

\section{References}

[Blockeel and Raedt 1998] Blockeel, H., and Raedt, L. D. 1998. Top-down induction of first-order logical decision trees. Artificial intelligence 101(1):285-297.

[Carlson et al. 2010] Carlson, A.; Betteridge, J.; Kisiel, B.; Settles, B.; Jr., E. H.; and T.Mitchell. 2010. Toward an architecture for never-ending language learning. In Proceedings of the Twenty-Fourth Conference on Artificial Intelligence (AAAI).

[Domingos and Lowd 2009] Domingos, P., and Lowd, D. 2009. Markov Logic: An Interface Layer for AI. San Rafael, CA: Morgan \& Claypool.

[Fung, Mangasarian, and Shavlik 2002] Fung, G.; Mangasarian, O.; and Shavlik, J. 2002. Knowledge-Based support vector machine classifiers. In NIPS, 01-09.

[Heckerman et al. 2001] Heckerman, D.; Chickering, D.; Meek, C.; Rounthwaite, R.; and Kadie, C. 2001. Dependency networks for inference, collaborative filtering, and data visualization. Journal of Machine Learning Research 49-75.

[Kunapuli et al. 2013] Kunapuli, G.; Odom, P.; Shavlik, J.; and Natarajan, S. 2013. Guiding an autonomous agent to better behaviors through human advice. In ICDM.

[Manning et al. 2014] Manning, C.; Surdeanu, M.; Bauer, J.; Finkel, J.; Bethard, S.; and McClosky, D. 2014. The Stanford CoreNLP natural language processing toolkit. In Proceedings of 52nd Annual Meeting of the Association for Computational Linguistics: System Demonstrations, 55-60.

[Meza-Ruiz and Riedel 2009] Meza-Ruiz, I., and Riedel, S. 2009. Jointly identifying predicates, arguments and senses using markov logic. In Proceedings of NAACL HLT.

[Mikolov et al. 2013] Mikolov, T.; Chen, K.; Corrado, G.; and Dean, J. 2013. Efficient estimation of word representations in vector space. Proceedings of Workshop at ICLR.

[Mikolov, Yih, and Zweig 2013] Mikolov, T.; Yih, W.; and Zweig, G. 2013. Linguistic regularities in continuous space word representations. Proceedings of NAACL HLT.

[Natarajan et al. 2010] Natarajan, S.; Khot, T.; Kersting, K.; Gutmann, B.; and Shavlik, J. 2010. Boosting relational dependency networks. In Proceedings of the International Conference on Inductive Logic Programming (ILP).

[Natarajan et al. 2014] Natarajan, S.; Picado, J.; Khot, T.; 
Kersting, K.; Re, C.; and Shavlik, J. 2014. Effectively creating weakly labeled training examples via approximate domain knowledge. In International Conference on Inductive Logic Programming.

[Neville and Jensen 2007] Neville, J., and Jensen, D. 2007. Relational dependency networks. In Introduction to Statistical Relational Learning. The MIT Press.

[Neville et al. 2003] Neville, J.; Jensen, D.; Friedland, L.; and Hay, M. 2003. Learning relational probability trees. In In Proceedings of the ACM International Conference on Knowledge Discovery and Data Mining (SIGKDD), 625630.

[Niu et al. 2011] Niu, F.; Ré, C.; Doan, A.; and Shavlik, J. W. 2011. Tuffy: Scaling up statistical inference in Markov logic networks using an RDBMS. Proceedings of Very Large Data Bases (PVLDB) 4(6):373-384.

[Odom et al. 2015a] Odom, P.; Bangera, V.; Khot, T.; Page, D.; and Natarajan, S. 2015a. Extracting adverse drug events from text using human advice. In Artificial Intelligence in Medicine (AIME).

[Odom et al. 2015b] Odom, P.; Khot, T.; Porter, R.; and Natarajan, S. 2015b. Knowledge-based probabilistic logic learning. In Twenty-Ninth AAAI Conference on Artificial Intelligence (AAAI).

[Riedel, Yao, and McCallum 2010] Riedel, S.; Yao, L.; and McCallum, A. 2010. Modeling relations and their mentions without labeled text. In Proceedings of the 2010 European conference on Machine learning and knowledge discovery in databases (ECML KDD).

[Roth et al. 2014] Roth, B.; Barth, T.; Chrupala, G.; Gropp, M.; and Klakow, D. 2014. Relationfactory: A fast, modular and effective system for knowledge base population. In Proceedings of the 14th Conference of the European Chapter of the Association for Computational Linguistics, EACL 2014, April 26-30, 2014, Gothenburg, Sweden, 89-92.

[Soni et al. 2016] Soni, A.; Viswanathan, D.; Pachaiyappan, N.; and Natarajan, S. 2016. A comparison of weak supervision methods for knowledge base construction. In 5th Workshop on Automated Knowledge Base Construction (AKBC) at NAACL.

[Surdeanu 2013] Surdeanu, M. 2013. Overview of the tac 2013 knowledge base population evaluation: English slot filling and temporal slot filling. In Proceedings of the Sixth Text Analysis Confernece (TAC 2013).

[Towell and Shavlik 1994] Towell, G., and Shavlik, J. 1994. Knowledge-based artificial neural networks. Artif. Intell. 70(1-2):119-165.

[Zhang et al. 2012] Zhang, C.; Niu, F.; Ré, C.; and Shavlik, J. 2012. Big data versus the crowd: Looking for relationships in all the right places. In Proceedings of the 50th Annual Meeting of the Association for Computational Linguistics: Long Papers - Volume 1, ACL '12, 825-834. Stroudsburg, PA, USA: Association for Computational Linguistics. 